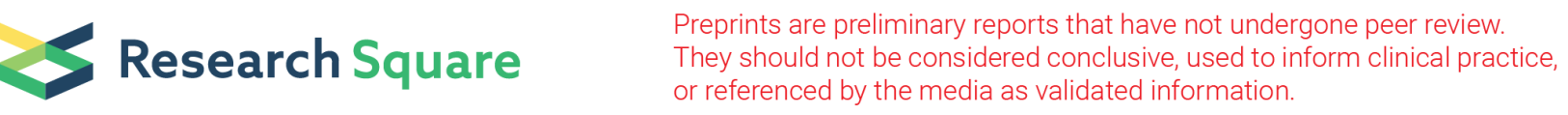

\title{
The Cumulative Therapeutic Effect of Acupuncture Treatment in Patients with Migraine without Aura: Evidence from Dynamic Alterations of Intrinsic Brain Activity and Effective Connectivity Analysis
}

Yilei Chen

Shuguang Hospital

Yingjie Kang

Shuguang Hospital

Shilei Luo

Shuguang Hospital

Shanshan Liu

Shuguang Hospital

Bo Wang

Shuguang Hospital

Zhigang Gong

Shuguang Hospital

Yanwen Huang

Shuguang Hospital

Hui Wang

Shuguang Hospital

WenLi Tan ( $\nabla$ tanying2245@163.com )

Shuguang Hospital https://orcid.org/0000-0001-7727-5772

Songhua Zhan

Shuguang Hospital

\section{Research Article}

Keywords: Migraine, Granger causality analysis, Amplitude of low-frequency fluctuations, Dynamic analysis, Acupuncture

Posted Date: January 12th, 2022

DOI: https://doi.org/10.21203/rs.3.rs-1055422/v2

License: (9) (1) This work is licensed under a Creative Commons Attribution 4.0 International License. Read Full License 


\section{Abstract}

We explored the dynamic alterations of intrinsic brain activity and effective connectivity after acupuncture treatment to investigate the underlying neurological mechanism of acupuncture treatment in patients with migraine without aura (MwoA). The fMRI scans were separately obtained at baseline, after the first and after the 12th acupuncture sessions in 40 patients with MwoA. Compared with HCs, patients with MwoA showed mostly decreased dynamic amplitude of low-frequency fluctuation (dALFF) variability in regions with differences. After acupuncture treatment, the decreased dALFF variability of the rostral ventromedial medulla (RVM), the superior lobe of left cerebellum (Cerebelum_Crus1_L), and the right precuneus (PCUN.R) progressively recovered. The RVM revealed gradually increased dynamic effective connectivity (DEC) variability outflow to the right middle frontal gyrus, the left insula, the right precentral gyrus, and the right supramarginal gyrus, and enhanced DEC variability from the right fusiform gyrus inflow to RVM. Furthermore, the increased DEC variability were found from Cerebelum_Crus1_L outflow to the left middle occipital gyrus and the left precentral gyrus, from PCUN.R outflow to the right thalamus. These dALFF variabilities were positive correlated with frequency of migraine attack and negative correlated with disease duration, dynamic GCA coefficients were positive correlated with Migraine-Specific Quality of Life Questionnaire score, negative correlated with frequency of migraine attack and visual analog scale score postacupuncture treatment. Our results provide insight into dynamic alterations from the perspective of dynamic local brain activity and effective connectivity for the understanding mechanisms of cumulative therapeutic effect of acupuncture in patients with MwoA.

\section{Introduction}

Migraine is a prevalent primary headache disorder characterized by recurrent headache attacks, nausea or vomiting, and sensitivity to sound or light [Headache Classification Committee of the International Headache Society (IHS), 2018]. There are two major types of migraine: migraine without aura (MwoA) and migraine with aura, MwoA is the most common type of migraine, patients with migraine usually have frequent, severe, and disabling headache attacks, which causes the enormous individual and social burden (Leonardi et al. 2005). Migraine is typically treated by various pharmacological or non-pharmacological therapies to relieve pain or prevent migraine attacks, but these methods have limited efficacy and multiple adverse effects, such as weight gain, fatigue, sleep disturbance, gastrointestinal reaction, and medication overuse (Diener et al. 2015). Acupuncture as one of the treatment modalities of Traditional Chinese Medicine (TCM) in china and is widely used as a complementary and alternative treatment to prevent migraine attacks and to relieve pain during a migraine, the long-term prophylactic effect of acupuncture for migraine is recognized (Xu et al. 2020; Zhao et al. 2017), however, the mechanism of cumulative effect of acupuncture treatment is currently unclear.

Recently, neuroimaging have provided significant new insights to explore the central mechanism of the effects of acupuncture on migraine (Chang et al. 2021; L. Liu et al. 2021). Several fMRI studies have indicated that verum and sham acupuncture have different modulation effects on amplitude of low-frequency fluctuation (ALFF) in the rostral ventromedial medulla (RVM)/trigeminocervical complex (TCC) of migraine patients (Li, Zeng, et al. 2017). Verum acupuncture elicited a more widespread and remarkable cerebral response, including the pain matrix, lateral pain system, medial pain system, default mode network, and cognitive components of pain processing compared to sham acupuncture (Zhao et al. 2014), Verum acupuncture also could normalize the abnormal network connectivity in the visual, default mode, sensorimotor, and frontal-parietal networks (Tu et al. 2020). Furthermore, it was demonstrated that acupuncture treatment could increased right frontoparietal network functional connectivity (Li, Lan, et al. 2017), and the connectivity of DMN had been normalized after acupuncture intervention (Zou et al. 2019). Nevertheless, these studies have been limited to compare the effect of acupuncture at pre- and post-treatment and not explored the continuous effects of acupuncture in different periods of treatment. Our previous studies demonstrated that acupuncture could improve the dysfunction of cerebellum, and activate brain regions involved in modulation of pain and emotion in patients with MwoA by the regional homogeneity (ReHo) analysis method (S. Liu et al. 2021). However, most of the studies above have focused on the static characterizations or traditional unidirectional functional connectivity (FC) of brain. Brain activity is inherently dynamic (Bassett \& Sporns 2017), Recently, a number of studies have proposed that brain activity was dynamically changed over time, so dynamic ALFF was an effective tool to explore brain dynamic activity in healthy people (Liao et al. 2019), the time-varying brain activity characterized by dynamic ALFF may underline the disruption of brain activity in various mental disorders (Fu et al. 2018), such as schizophrenia (Yang et al. 2019). Meanwhile, granger causality analysis (GCA) is a fMRI-based directed connectivity to assess causality between different brain regions, which can provide richer information for connectivity analysis than unidirectional FC (Huang et al. 2021; Wei et al. 2020). To date, the dynamic characteristics of ALFF and GCA have rarely been investigated the effect of acupuncture treatment in migraine patients. 
In the present study, patients with MwoA received 6 weeks of standard acupuncture treatment, and the fMRI scans were performed at baseline, after the first and 12th acupuncture sessions. Utilizing dynamic ALFF analysis method, we first explored the differences in dynamic ALFF variability between patients with MwoA and HCs at baseline. And then, the brain regions with intergroup differences were defined as the region of interests (ROI). Dynamic ALFF and GCA analysis method were employed to explore the standard deviation (SD) value of the dALFF and GCA variability of different time periods after acupuncture treatments. Finally, correlation analysis were performed to investigate the associations between the values of dALFF and GCA variability and clinical variables. In this study, we hypothesized that (1) there would be several differences in dynamic ALFF at baseline inter-group comparison, (2) the dALFF and GCA variability may be alter during the different periods of acupuncture treatment, (3) these alterations would be associated with clinical features.

\section{Materials And Methods}

\section{Participants}

Forty patients with MwoA were enrolled from outpatient in department of neurology or acupuncture at the Shuguang Hospital affiliated to Shanghai University of Traditional Chinese Medicine. Thirty-six aged-matched, education level-matched with patients and righthanded healthy controls (HCs) were recruited. All participants signed written informed consent. This trial was approved by the Ethics Committee of the Shuguang Hospital affiliated to Shanghai University of Traditional Chinese Medicine and is registered on www.chictr.org.cn (ChiCTR1900023105). The diagnosis of MWoA was established according to the International Classification of Headache Disorders, 3rd Edition ICHD-III criteria ("Headache Classification Committee of the International Headache Society (IHS) The International Classification of Headache Disorders, 3rd edition" 2018). Inclusion criteria required that all patients: 1) were 18-65 years old and right-handed; 2) had two to eight times of migraine attacks during the past month; 3 ) had at least six months of migraine history; and 4) had no prophylactic headache medications during the past month, had no psychoactive or vasoactive agents during the last three months. The exclusion criteria included the following: 1) suffered from other type of primary or secondary headache; 2 ) had a history of head trauma or brain tumor; 3) had any other neurological or psychiatric disorder; 4) were pregnancy or breast-feeding; 5) had MRI or acupuncture contraindications.

\section{Study design}

The total observation period for Patients with MwoA of this study was ten weeks. Weeks 1 to 4 served as a baseline phase,and all patients had recorded headache diaries at baseline. Weeks 5-10 served as an intervention phase. During this period, patients with MwoA standard acupuncture treatment. All the patients maintained a headache diary during the study period. FMRI scans were administered before and immediately after the first and 12th acupuncture sessions for patients with MwoA (all fMRI were scanned within $1 \mathrm{~h}$ before and after acupuncture). All patients with MwoA had been migraine-free for at least $72 \mathrm{~h}$ at the time of the fMRI scans. HCs group only received the baseline MRI scan (see Fig. 1).

\section{Acupuncture treatment}

In our study, the patients with MwoA were performed 12 sessions of acupuncture (twice a week, finished in 6 weeks), and every session lasted for 20 minutes. Acupoints were selected according to the standardized acupuncture protocol: Baihui (DU20), Taiyang (EX-HN5), bilateral Fengchi (GB20), Shuaigu (GB8), Xuanlu (GB5), Toulinqi (GB15), Hegu(LI4), and Taichong (LR3) (Zhao et al. 2017). Two licensed acupuncturists (Wang B and Liu S) were responsible for all the acupuncture treatments. Sterile disposable acupuncture needles of $25-40 \mathrm{~mm}$ in length and $0.25 \mathrm{~mm}$ in diameter were inserted to achieve the sensation of deqi. Electrical stimulation was applied bilaterally at GB20 and GB8 at a frequency of $2 \mathrm{~Hz}$ and intensity ranging from 0.1 to $1.0 \mathrm{~mA}$ until the patient felt bearable. All participants agreed not to take any conventional medication for migraine during the study period. In cases of severe pain, ibuprofen (as $300 \mathrm{mg}$ extended-release capsules) was allowed as a rescue medication.

\section{Clinical assessments}

During the four weeks before the first fMRI scans and after all the acupuncture sessions, the frequency of migraine attack (days/month), VAS (0-10 scale, 10 being the most intense imaginable pain), Self-Rating Anxiety Scale (SAS), Self-Rating Depression Scale (SDS) and MSQ were assessed. Adverse events associated with acupuncture, including bleeding, subcutaneous bleeding, severe pain, fainting and local infection, were recorded at each treatment. 


\section{Data acquisition}

MR scans were acquired on a 3.0-T MRI scanner (UMR780 Platform, United Imaging Medical Systems, Shanghai, China) with an 12channel flexible head coil at the Shuguang Hospital MRI Center. The rest fMRI images were obtained axially by a multislice gradientecho echo-planar imaging (EPI) sequence and the parameters were as following: repetition time (TR)=2000 ms, echo time (TE)=30 ms, flip angle $=90^{\circ}$, field of view $=240 \times 240 \mathrm{~mm}^{2}$, matrix $=64 \times 64,33$ contiguous slices with $3.5 \mathrm{~mm}$ slice thickness, 240 time points. Structural images were acquired by a three-dimensional turbo fast echo (3D-TFE) sequence with voxel size of $1 \mathrm{~mm}^{3}$, and the parameters were as following: $T R=7.2 \mathrm{~ms}, T E=3.1 \mathrm{~ms}$, slice thickness, $1.0 \mathrm{~mm}$, flip angle $=10^{\circ}$, field of view $=256 \times 256 \mathrm{~mm}^{2}$, matrix $=256 \times 256,176$ slices without interslice gap. A cushion was placed into the coil to fix the head and reduce motion. The participants were instructed to keep still with eyes close, to relax but not to fall asleep, and to try not to think about anything.

\section{Data preprocessing}

FMRI data preprocessing was performed by DPABI software (http://www.rfmri.org/) in MATLAB. The preprocessing course consisted of the following steps: 1) the first 10 images were discarded and the remained 230 images were used for data analysis; 2) slice timing correction; 3) head motion correction (the translation or rotation motion in any given data did not exceed $2.0 \mathrm{~mm}$ or $2.0^{\circ}$ ); 4) The coregistered functional images were spatially normalized to the Montreal Neurological Institute (MNI) space and resampled to 3-mm cubic voxels; 5) linear trend removal was performed to reduce the effect of low-frequency drifts; 6) nuisance covariates regression (the white matter signal, the cerebrospinal fluid signal and 24 head motion parameters; 7) lintemporal band-pass filtering at a frequency band of $0.01-0.08 \mathrm{~Hz}$. After these head motion controls, eleven of the subjects (three HCs and eight patients with MwoA) were excluded.

\section{Dynamic ALFF analysis}

The dynamic ALFFfor each participant was performed by DynamicBC (v2.2 www.restfmri.net/forum/DynamicBC) toolbox. Specifically, a temporal rectangular window was firstly chosen. Then, the ALFF values in each window were calculated. Window length was an important parameter in resting-state dynamics computation. The 'rule of thumb' of sliding-window length is that the minimum window length should be no less than $1 / \mathrm{fmin}$, fmin $=0.01 \mathrm{~Hz}$. Here, a window length of $50 \mathrm{TR}$ was considered as the optimal parameter to maintain the balance between capturing a rapidly shifting dynamic relationship and obtaining reliable estimates of the correlations between regions (Cui et al. 2020; Li et al. 2019), The sliding window was systematically shifted with a step size of 5 five TR (10 s) to calculate the dALFF of each participant. The preprocessed data of each individual were segmented into 37 windows, and the ALFF map was obtained for each sliding window. Subsequently, we measured the variance of these maps using standard deviation (SD) to evaluate the temporal variability of dALFF across 37 windows. The dALFF variability of each voxel was further transformed into a zscore by subtracting the mean and dividing by the SD of global values. Finally, the mean normalized dALFF maps were spatially smoothed using an isotropic Gaussian kernel of $8 \mathrm{~mm}$ full-width at half-maximum.

\section{Dynamic effective connectivity analysis}

In this study, we performedseed-baseddynamic Granger causality analysis (GCA) by the DynamicBC toolbox to detect the dynamic effective connectivity (DEC). The time series of the each ROI based on dALFF results was defined as the seed time series $X$, and the time course of voxels within the whole brain was defined as Y. A bivariate coefficient GCA to investigate the Granger causal influence between the per $\mathrm{ROI}$ and each voxel of the whole brain. A positive coefficient indicates that activity in region $\mathrm{X}$ exerts a positive influence on activity in region $Y$, whereas a negative coefficient indicated that the activity of region $X$ exerted a negative influence on the activity of region $Y$. The dynamic GCA was estimated using the sliding window approach above, the time series of each participant was also divided into 37 windows. Thus, For each subject, the averaged time course of GCA coefficient of each ROI was extracted across 37 windows and concatenated to form a $2 \times \mathrm{W} \times \mathrm{N}$ matrix (where $\mathrm{W}$ denotes the number of windows and $\mathrm{N}$ denotes the number of ROIs). The DEC variability for each ROI was assessed with the SD of the averaged time course of GCA coefficient across 37 windows. Finally, the dynamic GCA coefficient maps for all subjects were then converted to z-scores by Fisher z-transformation.

\section{Statistical analyses}

Demographic characteristics were evaluated between MwoA and HCs. Differences of two groups in age and education level were analyzed with two-sample $t$ test; $\chi 2$ test was used for analyzing the difference of gender in two groups. Two-sample $t$-tests or Mann- 
Whitney $U$ test was used to compare differences in the clinical variables between the two time points. P $<0.05$ existed statistical difference.

Two-sample t-tests were performed to compare dALFF variability maps between MwoA at baseline and HCs within a gray matter mask with age, gender, education and head motion as covariates. The resultant T-maps were corrected for multiple comparisons using the Gaussian random field (GRF) theory (voxel $p<0.001$, cluster $p<0.05$, two tailed).

To find the differences effect of acupuncture during the different periods of treatment, we first performed repeated-measures one-way ANOVA to investigate the dALFF variability among the different periods. The SD value of each brain region with significant difference between groups was extracted for statistical analysis in SPSS version 25.0 (SPSS, Inc., Chicago, IL, United States), and post hoc t-tests were performed to detect differences of dALFF variability among two periods (false discovery rate corrected, $P<0.05$ ).

For group level analyses on DEC of the ROIs, the SD values of Zx $\rightarrow$ y and Zy $\rightarrow x$ dynamic GCA coefficient maps were calculated for each group. These maps were entered into repeated-measures one-way ANOVA to determine the difference among the different periods with age, sex, and education level included as covariates. Multiple comparison correction was performed based on Gaussian random field theory (GRF, voxelwise $p<0.001$, cluster-wise $p<0.05$, two-tailed). Post hoc t-tests were performed to detect the differences in DEC variability between two periods (false discovery rate corrected, $P<0.05$ ).

Finally, the SD value of the dALFF variability and DEC variability in regions with significant differences in each MwoA individual were extracted, Based on these regions, Pearson/Spearman correlation was analyzed to probe the relation of alterations in dALFF variability/DEC variability to the clinical data of MwoA. The significance was set at a threshold of $p<0.05$ using Bonferroni correction.

\section{Validation analyses}

To validate the main findings of dALFF variability and DEC variability obtained from sliding-window length of 50 TR, we carried out auxiliary analyses with different sliding window lengths(30 and 80 TR).

\section{Results}

\section{Demographic and clinical characteristics at baseline}

The demographic information and clinical characteristics of all the participants are presented in Table 1 . There were no statistical difference in age $(p=0.408)$, education level $(p=0.313)$, and gender $(p=0.490)$ between patients with MwoA and HCs. The disease duration of the MwoA patients group was $16.21 \pm 12.56$ years, the frequency of migraine attack was $5.14 \pm 1.53$ days, and the VAS score was $7.81 \pm 1.39$ (see Table 1).

Table 1

Demographic and clinical characteristics of patients with migraine without aura and healthy controls at baseline

\begin{tabular}{|lllc|}
\hline Characteristics & migraine patients at baseline(n=40) & HCs(n=33) & P-value \\
\hline Age (years) & $38.02 \pm 9.79$ & $33.26 \pm 5.76$ & 0.408 \\
\hline Gender (male/female) & $6 / 34$ & $7 / 26$ & 0.490 \\
\hline Education (years) & $15.02 \pm 3.19$ & $15.76 \pm 1.76$ & 0.313 \\
\hline Disease duration(years) & $16.21 \pm 12.56$ & - & - \\
\hline Frequency of migraine attack(days) & $5.14 \pm 1.53$ & - & - \\
\hline VAS score & $7.81 \pm 1.39$ & - & - \\
\hline
\end{tabular}

\section{Differences in dynamic ALFF analysis between patients with MwOA at baseline and HCs}

The brain areas with statistical difference in dynamic ALFF variability were shown in Table 2. Compared with HCs, patients with MwoA at baseline showed decreased dALFF variability in the Rostral ventromedial medulla (RVM), Superior lobe of left cerebellum ( Cerebelum_Crus1_L), the right inferior frontal gyrus, triangular part (IFGtriang. R), right Median cingulate, paracingulate gyri (DCG.R), 
right Precuneus (PCUN.R), left Inferior parietal, supramarginal and angular gyri (IPL.L). Conversely, compared with HCs, patients with MwoA showed increased dALFF variability only in the left Inferior occipital gyrus (IOG.L).

Table 2

Brain regions with increased and decreased dALFF variability in migraine compared with healthy controls at baseline

\begin{tabular}{|lllll|}
\hline Contrast & Brain regions & peak MNI $(\mathbf{x}, \mathbf{y}, \mathbf{z})$ & voxels & T score \\
\hline MwoA < HCs & RVM & $0-28-46$ & 45 & -3.77 \\
\cline { 2 - 4 } & Cerebelum_Crus1_L & $-30-69-30$ & 32 & -3.39 \\
\hline IFGtriang.R & 422115 & 39 & -4.67 \\
\hline DCG.R & 61236 & 21 & -5.16 \\
\hline PCUN.R & $9-5754$ & 26 & -4.80 \\
\hline MwoA > HCs & IPL.L & $-36-6354$ & 37 & -4.45 \\
\hline
\end{tabular}

Gaussian Random Field theory correction, voxel $\mathrm{P}$ value $<0.001$, cluster $\mathrm{P}$ value $<0.05$; All abbreviations are defined in the Abbreviations section.

\section{Clinical Outcomes}

After 12 sessions of acupuncture treatment, the frequency of migraine attack and the VAS score were significantly lower than those at baseline $(p<0.001)$. The SAS scores, SDS scores and MSQ scores (restrictive, preventive, and emotional functional subscales) were significant improved $(p<0.001)$ (see Table 3$)$.

Table 3

Clinical outcomes in patients with migraine without aura during the study period( $\mathrm{n}=29)$

\begin{tabular}{|lllllllll|}
\hline $\begin{array}{l}\text { Assessment } \\
\text { points } \\
\text { (wks) }\end{array}$ & $\begin{array}{l}\text { Frequency } \\
\text { of migraine } \\
\text { attack(days) }\end{array}$ & VAS score & SAS score & SDS score & $\begin{array}{l}\text { MSQ score } \\
\text { (restrictive } \\
\text { subscale) }\end{array}$ & $\begin{array}{l}\text { MSQ score } \\
\text { (preventive } \\
\text { subscale) }\end{array}$ & $\begin{array}{l}\text { MSQ score } \\
\text { (emotional } \\
\text { subscale) }\end{array}$ & $P$-value \\
\hline At baseline & $5.14 \pm 1.53$ & $7.81 \pm 1.39$ & $46.04 \pm 7.03$ & $49.68 \pm 8.29$ & $58.24 \pm 15.65$ & $60.89 \pm 21.27$ & $63.46 \pm 23.05$ & $P<0.001$ \\
\hline $\begin{array}{l}\text { At } \\
\text { Treatment }\end{array}$ & $1.85 \pm 1.32$ & $3.67 \pm 1.20$ & $38.41 \pm 8.03$ & $39.38 \pm 9.87$ & $80.33 \pm 10.36$ & $85.39 \pm 13.22$ & $87.67 \pm 16.37$ & \\
\hline
\end{tabular}

\section{Dynamic ALFF analysis during the different periods of treatment in patients with MwoA}

The seven differential brain regions in dynamic ALFF analysis above were extracted as the ROls. According to repeated-measures oneway ANOVA tests, we found that the dALFF variability were significant different in RVM, Cerebelum_Crus1_L, and PCUN.R. Post hoc tests revealed that the dALFF variability of patients with MwoA increased significantly in the RVM after the first acupuncture session compared with at baseline, after all sessions of acupuncture compared with at baseline and after the first acupuncture session. The dALFF variability of the Cerebelum_Crus1_L and PCUN.R after all sessions of acupuncture was significantly increased compared with after the first acupuncture session. There were no statistical differences in the dALFF variability within the other ROIs (see Fig. 2).

\section{Seed-based dynamic GCA analysis during the different periods of treatment in patients with MwoA}

The bivariate RVM-to-whole-brain dynamic GCA showed that DEC variability from RVM outflow to the right Middle frontal gyrus (MFG.R), the left Insula (INS.L), the right Precentral gyrus (PreCG.R), and the right Supramarginal gyrus (SMG.R) were significantly enhanced in repeated-measures one-way ANOVA tests (see Table 4). Post hoc tests revealed that the DEC variability from RVM outflow to INS.L and PreCG.R after the first acupuncture session were enhanced compared with at baseline. The DEC variability from RVM outflow to MFG.R, INS.L, PreCG.R, and SMG.R were significantly enhanced after all sessions of acupuncture compared with at baseline and after the first acupuncture session. Next, whole-brain-to-RVM dynamic GCA showed that the DEC variability from the right Fusiform 
gyrus (FFG.R) inflow to RVM was significantly enhanced. Post hoc tests revealed that the DEC variability from FFG.R to RVM after all sessions of acupuncture was significantly increased compared with after the first acupuncture session (see Fig. 3). Furthermore, the bivariate Cerebelum_Crus1_L-to-whole-brain dynamic GCA showed that DEC variability from Cerebelum_Crus1_L outflow to the left Middle occipital gyrus (MOG.L) and the left Precentral gyrus (PreCG.L) (see Table 4) were significantly enhanced. Post hoc tests indicated that the DEC variability after all sessions of acupuncture significantly enhanced compared with at baseline and after the first acupuncture session, while there was no significant difference in DEC variability after the first acupuncture session compared with at baseline (see Fig. 4). In addition, we also observed that the bivariate PCUN.R-to-whole-brain dynamic GCA displayed significantly enhanced DEC variability from PCUN.R outflow to the right Thalamus (THA.R) (see Table 4), and post hoc tests indicated that the DEC variability after the first acupuncture session was enhanced compared with at baseline, after all sessions of acupuncture compared with at baseline and after the first acupuncture session (see Fig. 5). There were no statistical difference in DEC variability in the remained ROIs.

Table 4

The DEC variability significantly during the different periods of treatment

\begin{tabular}{|c|c|c|c|c|}
\hline Contrast & Brain region & peak MNI $(x, y, z)$ & voxels & F score \\
\hline \multirow{2}{*}{ Causal outflow from RVM to the rest of the brain ( $\mathrm{X}$ to $\mathrm{Y}$ ) } & SMG.R & $57-1818$ & 19 & 6.39 \\
\hline & PreCG.R & 57321 & 21 & 8.75 \\
\hline Causal outflow from Cerebelum_Crus1_L & MOG.L & $-33-6630$ & 16 & 6.89 \\
\hline to the rest of the brain ( $\mathrm{X}$ to $\mathrm{Y}$ ) & PreCG.L & -39639 & 18 & 8.44 \\
\hline Causal outflow from Precuneus_R to the rest of the brain ( $X$ to $Y$ ) & THA.R & $3-180$ & 19 & 8.91 \\
\hline
\end{tabular}

Correlation between dALFF variability, DEC variability and clinical variables at baseline and after acupuncture treatment

The SD value of the dALFF variability in RVM within patients with MwoA were significantly positive correlated with frequency of migraine attack and negative correlated with disease duration at baseline $(p<0.001, r=0.597 ; p=0.033, r=-0.338$ respectively). The frequency of migraine attack was also significantly positive correlated with the SD value of the dALFF variability in Cerebelum_Crus1_L ( $p=0.049, r=0.314$ ) (see Fig. S1). The SD value of the dynamic GCA coefficient between MFG.R, INS.L, PreCG.R, and SMG.R, and FFG.R with RVM were significantly positive correlated with MSQ score, negative correlated with frequency of migraine attack and VAS score (see Fig. S2 and Table 5) during the different periods of treatment. In addition, there were significant negative correlations between the SD value of the dynamic GCA coefficient from Cerebelum_Crus1_L to MOG.L and PreCG.L with VAS score $(p=0.006, r=$ $-0.360 ; p=0.009, r=-0.340$ respectively), and also was significant negative correlations between the from Cerebelum_Crus1_L to PreCG.L with frequency of migraine attack ( $p=0.002, r=-0.397)$ (see Fig. S3). Moreover, the VAS score and frequency of migraine attack were significant negative correlations with the SD value of the dynamic GCA coefficient from PCUN.R to THA.R $(p<0.001, r=$ $-0.544 ; p<0.001, r=-0.691$ respectively), and were significantly positive correlated with MSQ score $(p<0.001, r=-0.568 ; p<0.001, r=$ $-0.675 ; p<0.001, r=-0.566$ respectively) (see Fig. S4). No other significant linear correlation was observed. 
Table 5

The values of the dynamic GCA coefficient between MFG.R, INS.L, PreCG.R, and SMG.R, and FFG.R with RVM

\begin{tabular}{|llllll|}
\hline ROIs & $\begin{array}{l}\text { Frequency of migraine } \\
\text { attack (days) (r,p-value) }\end{array}$ & $\begin{array}{l}\text { VAS score } \\
(r, p \text {-value) }\end{array}$ & $\begin{array}{l}\text { MSQ score(restrictive } \\
\text { subscale)(r,p-value) }\end{array}$ & $\begin{array}{l}\text { MSQ score(preventive } \\
\text { subscale)(r,p-value) }\end{array}$ & $\begin{array}{l}\text { MSQ score(emotional } \\
\text { subscale)(r,p-value) }\end{array}$ \\
\hline INS.L & $-0.515,<0.001$ & $\begin{array}{l}-0.508,< \\
0.001\end{array}$ & $0.424,<0.001$ & $0.412,0.001$ & $0.453,<0.001$ \\
\hline MFG.R & $-0.387,0.004$ & $\begin{array}{l}-0.398, \\
0.002\end{array}$ & $0.408,0.002$ & $0.394,0.005$ & $0.422,<0.001$ \\
\hline PreCG.R & $-0.406,0.002$ & $\begin{array}{l}-0.544,< \\
0.001\end{array}$ & $0.468,<0.001$ & $0.530,<0.001$ & $0.508,<0.001$ \\
\hline SMG.R & $-0.534,<0.001$ & $\begin{array}{l}-0.323, \\
0.013\end{array}$ & $0.455,<0.001$ & $0.527,<0.001$ & $0.359,0.006$ \\
\hline FFG.R & $-0.470,<0.001$ & $\begin{array}{l}0.384 \\
\text { All abbreviations are defined in the Abbreviations section. }\end{array}$ & $0.429,<0.001$ & $0.340,0.009$ \\
\hline
\end{tabular}

\section{Validation Results}

To verify the stability of our main results, other window sizes were included, such as 30 TRs and 80 TRs. The analysis of dynamic ALFF analysis and dynamic DEC analysis using different sliding-window lengths supported our main results (see Fig. S5-S8).

\section{Discussion}

In this study, we applied the dALFF analysis to assess the abnormal of variability between in patients with MwoA and HCs. And the changes of dALFF and GCA variability in the follow-up phase after acupuncture treatments were found. These alterations were associated with clinical variables. The results could be summarized as follows: (1) most of the differential brain regions of dALFF variability were decreased compared with HCs at baseline, included RVM, Cerebelum_Crus1_L, and PCUN.R etc. (2) the decreased dALFF variability of brain regions of RVM, Cerebelum_Crus1_L, and PCUN.R progressively recovered and the DEC was variability gradually increased after acupuncture treatment. (3) these dALFF variabilities were positively correlated positively with the frequency of migraine attacks and negatively with the disease duration at baseline, and the dynamic GCA coefficients were positively correlated positively with MSQ scores, and negatively with the frequency of migraine attacks and VAS scores after postacupuncture treatment. Overall, our findings proved that the cumulative therapeutic effects of acupuncture treatment in patients with MwoA were related with the changes of the dynamic local brain activity and effective connectivity.

We firstly found an decreased dALFF variability at the brain region of RVM in patients with migraine without aura. The ALFF has been proven to be an effective and reliable parameter for evaluating local intrinsic brain activity (Zang et al. 2007), As an extensive index of ALFF, the dynamic ALFF analysis subdivided the whole time series into multiple slices and then calculated ALFF in each slice. RVM was a part of brainstem, and a critical region of the descending pain modulatory system (Kong et al. 2010), ON and OFF cells within the RVM were activated on the onset and offset of noxious stimulation (Fields 2004). Result of RVM was similar to the study demonstrated that Patients with MwoA showed increased ALFF in posterior insula and putamen/caudate, and reduced ALFF in RVM/ trigeminocervical complex (TCC) (Li, Zeng, et al. 2017), in contrast to another study that migraineurs had significant fractional amplitude of low-frequency fluctuation (fALFF) increases in bilateral ventral posteromedial(VPM) thalamus and brainstem encompassing RVM and TCC (Kim et al. 2021). Inconsistencies in results for RVM might be attributed to the different subtypes of migraine, different sample size, and methodological variability. After acupuncture treatment, we also found that the decreased dALFF variability of the RVM progressively recovered, and this finding is comply with many studies reporting that acupuncture treatment could normalize the impaired descending pain modulatory system in migraine (Li et al. 2016; Li, Zeng, et al. 2017). Go further, the DEC variability from RVM outflow to INS.L and PreCG.R after the first acupuncture session were enhanced compared with at baseline, outflow to MFG.R, INS.L, PreCG.R, and SMG.R and inflow to RVM from FFG.R were enhanced after all sessions of acupuncture compared with the first acupuncture session. The insula may be associated with the different aspects of sensorimotor and cognitive control of speech production(Battistella et al. 2018; Schwedt et al. 2015). The precentral gyrus is the traditional movement-related higher cortex and includes the primary motor area, the somatomotor cortex and the lateral premotor area(Arca et al. 2021; Meier et al. 
2008). The prefrontal cortex is the central brain region for executive functions(Ashina et al. 2021; Ueda et al. 2018). The fusiform gyrus is responsible for processing colour information, face and body recognition and the emotional expression of facial stimuli(Bassez et al. 2020; Parvizi et al. 2012). These can indicate that the acupuncture treatment can gradually affect the cognitive control, sensorimotor and speech functions of patients with migraine without aura through the RVM. This study also showed that the dALFF variability in RVM was correlated with frequency of migraine attack and disease duration, these DEC variabilities of the RVM to-whole-brain were correlated with frequency of migraine attack, disease duration, and MSQ scores after postacupuncture treatment, suggested that these brain regions might be one of the therapeutic targets.

Our results additionally showed the decreased dALFF variability in the Cerebelum_Crus1_L, IFGtriang.R, DCG.R, PCUN.R, and IPL.L. These regions were parts of the default, executive control, and cerebellar networks (Kim et al. 2017; Tu et al. 2019). A recent study revealed the abnormal dynamic low-frequency oscillation in default mode network, salience network, and executive control network in migraine patients (Chen et al. 2021), Another study showed the decreased ALFF value in the bilateral cerebellum posterior lobe, left cerebellum anterior lobe, bilateral orbital cortex, right middle frontal gyrus, bilateral occipital lobe, right fusiform gyrus, and bilateral postcentral gyrus in patients with migraine (Wang et al. 2016). These studies were generally consistent with the results of this study revealed that the pain related networks in migraine.

In addition, the dALFF variability of the Cerebelum_Crus1_L and PCUN.R were also slowly enhanced following treatment. Many studies have demonstrated the preventive effect of acupuncture on migraine might be through modulation of the visual network, the default mode network (DMN), the sensorimotor network, the frontal lobe network (FPN) and the descending pain modulation system(Tian, Guo, et al. 2021; Tian, Yin, et al. 2021; Tu et al. 2020; Zou et al. 2019), these are similar to the results of this study, and the dALFF variability in Cerebelum_Crus1_L was correlated with frequency of migraine attack. Furthermore, acupuncture treatment was a long-term and cumulative process (Zhao et al. 2017). Most of the fMRI studies of acupuncture on migraine focused only on pre- and post-treatment changes, And the changes in the whole process of acupuncture treatment were unknown. Thus, in our current study, the changes of dALFF variability at three time points with acupuncture treatment in patients with MwoA by the perspective of local brain activity demonstrated that the cumulative therapeutic effect of acupuncture. Moreover, the study additionally showed that the enhanced DEC variability outflow from Cerebelum_Crus1_L to the left Middle occipital gyrus and the left Precentral gyrus, and outflow from PCUN.R to the right thalamus enhanced gradually after the process of acupuncture treatment. The Middle occipital gyrus is an important component of the visual network, responsible for the afferent input, integration and perception of visual information(Zhu et al. 2018). Previous studies demonstrated a transient pathologic state with atypical thalamo-cortical connectivity in migraineurs(Coppola et al. 2021). These results be able to intuitively explain the cumulative therapeutic effect of acupuncture treatment. It was also shown that the cumulative therapeutic effects of acupuncture treatment in migraine could be regarded as the consequence of interactions between pain modulation and cortical networks, which might provide an objective reference of treatment in migraine. Moreover, these DEC variabilities of Cerebelum_Crus1_L, and PCUN.R to-whole-brain were correlated with frequency of migraine attack, disease duration, and MSQ scores after postacupuncture treatment, which suggested that these brain regions might be the target areas for acupuncture recovering migraine without aura.

Our study has several potential limitations. First, there was no follow-up in the control group, which made it impossible to evaluate the interaction between the groups and time. However, all patients with MwoA performed a series of fMRI scans at three time points, which enabled to get the longitudinal evidence of cumulative therapeutic effect in acupuncture treatments. Second, the optimal sliding window length to obtain dynamic changes in brain activity remains unclear. We selected 50 TRs as the window length based on previous studies and validated our results by using different sliding window lengths and demonstrated that our findings were stable and not influenced by this factor. Third, the small sample size may have weakened the liability of our results and they should be proved by the study with a big sample size in the future.. Fourth, multimodal brain imaging (i.e. diffusion tensor imaging) was useful for studying anatomical connectivity of patients with MwoA after acupuncture treatments, and it could be employed in experiments in the future.

\section{Conclusion}

In conclusion, this prospective longitudinal study showed that the cumulative therapeutic effect of acupuncture in Patients with MwoA manifested as the impaired brain regions of local brain activity progressively recovered and enhanced effective connectivity with other brain regions gradually from the perspective of dynamic local brain activity and effective connectivity, which could help enhance our understanding of the mechanism of acupuncture for migraine. 


\section{Abbreviations}

MwoA: migraine without aura; dALFF: dynamic amplitude of low-frequency fluctuation; GCA: dynamic granger causality analysis; DEC: dynamic effective connectivity; ReHo: regional homogeneity; RVM: rostral ventromedial medulla; Cerebelum_Crus1_L: superior lobe of left cerebellum; IFGtriang.R: right inferior frontal gyrus, triangular part; DCG.R: right median cingulate and paracingulate gyri; PCUN.R: right precuneus; IPL.L: left inferior parietal, supramarginal and angular gyri; IOG.L: left inferior occipital gyrus; MFG.R: right middle frontal gyrus; INS.L: left insula; PreCG.R: right precentral gyrus; SMG.R: right supramarginal gyrus; FFG.R:right fusiform gyrus; MOG.L: left middle occipital gyrus; PreCG.L: left precentral gyrus; THA.R: right thalamus; MSQ: Migraine-Specific Quality of Life Questionnaire; VAS: visual analog scale; SDS: Self-Rating Depression Scale; TCM: Traditional Chinese Medicine; FC: functional connectivity; fMRI: Functional Magnetic Resonance Imaging; ROIs: region of interests; SD: standard deviation; healthy controls(HCs); GRF: gaussian random field.

\section{Declarations}

Acknowledgments The authors would like to thank all the patients with migraine without aura and healthy controls who participated in our research.

Author contributions WT and SZ designed the study. YC, YK, SL, SL, BW, ZG, YH, and HW performed the experiments. YC analysed the data and was major contributor in writing the manuscript. All authors read and approved the final manuscript.

Funding This work was supported by funding from Shanghai Science and Technology Committee (No. 18401970900, No. 18401970300) and Shanghai Health Care Commission Project (No. 202040194).

Conflict of interest No conflicts of interest to declare.

Ethical approval The studies were reviewed and approved by the Ethics Committee of the Shuguang Hospital affiliated to Shanghai University of Traditional Chinese Medicine.

Informed consent Informed consent was obtained from all participants included in the study.

\section{References}

1. Arca, K. N., VanderPluym, J. H., \& Halker Singh, R. B. (2021). Narrative review of neuroimaging in migraine with aura. Headache. https://doi.org/10.1111/head.14191.

2. Ashina, S., Bentivegna, E., Martelletti, P., \& Eikermann-Haerter, K. (2021). Structural and Functional Brain Changes in Migraine. Pain Ther, 10(1), 211-223. https://doi.org/10.1007/s40122-021-00240-5.

3. Bassett, D. S., \& Sporns, O. (2017). Network neuroscience. Nature Neuroscience, 20(3), 353-364. https://doi.org/10.1038/nn.4502.

4. Bassez, I., Van de Steen, F., Ricci, K., Vecchio, E., Gentile, E., Marinazzo, D., \& de Tommaso, M. (2020). Dynamic Causal Modelling of the Reduced Habituation to Painful Stimuli in Migraine: An EEG Study. Brain Sci, 10(10). https://doi.org/10.3390/brainsci10100712.

5. Battistella, G., Kumar, V., \& Simonyan, K. (2018). Connectivity profiles of the insular network for speech control in healthy individuals and patients with spasmodic dysphonia. Brain Struct Funct, 223(5), 2489-2498. https://doi.org/10.1007/s00429-0181644-y.

6. Chang, C. M., Yang, C. P., Yang, C. C., Shih, P. H., \& Wang, S. J. (2021). Evidence of Potential Mechanisms of Acupuncture from Functional MRI Data for Migraine Prophylaxis. Curr Pain Headache Rep, 25(7), 49. https://doi.org/10.1007/s11916-021-00961-4.

7. Chen, H., Qi, G., Zhang, Y., Huang, Y., Zhang, S., Yang, D., . . Zeng, M. (2021). Altered Dynamic Amplitude of Low-Frequency Fluctuations in Patients With Migraine Without Aura. Front Hum Neurosci, 15, 636472. https://doi.org/10.3389/fnhum.2021.636472.

8. Coppola, G., Di Renzo, A., Tinelli, E., Petolicchio, B., Parisi, V., Serrao, M., . . Pierelli, F. (2021). Thalamo-cortical networks in subtypes of migraine with aura patients. J Headache Pain, 22(1), 58. https://doi.org/10.1186/s10194-021-01272-0.

9. Cui, Q., Sheng, W., Chen, Y., Pang, Y., Lu, F., Tang, Q., . . Chen, H. (2020). Dynamic changes of amplitude of low-frequency fluctuations in patients with generalized anxiety disorder. Hum Brain Mapp, 41(6), 1667-1676. https://doi.org/10.1002/hbm.24902

Page 10/16 
10. Diener, H.-C., Charles, A., Goadsby, P. J., \& Holle, D. (2015). New therapeutic approaches for the prevention and treatment of migraine. The Lancet Neurology, 14(10), 1010-1022. https://doi.org/10.1016/s1474-4422(15)00198-2.

11. Fields, H. (2004). State-dependent opioid control of pain. Nat Rev Neurosci, 5(7), 565-575. https://doi.org/10.1038/nrn1431.

12. Fu, Z., Tu, Y., Di, X., Du, Y., Pearlson, G. D., Turner, J. A., . . Calhoun, V. D. (2018). Characterizing dynamic amplitude of lowfrequency fluctuation and its relationship with dynamic functional connectivity: An application to schizophrenia. Neuroimage, 180(Pt B), 619-631. https://doi.org/10.1016/j.neuroimage.2017.09.035.

13. Headache Classification Committee of the International Headache Society (IHS) The International Classification of Headache Disorders, 3rd edition. (2018). Cephalalgia, 38(1), 1-211. https://doi.org/10.1177/0333102417738202.

14. Huang, X., Zhang, D., Wang, P., Mao, C., Miao, Z., Liu, C., . . Wu, X. (2021). Altered amygdala effective connectivity in migraine without aura: evidence from resting-state fMRI with Granger causality analysis. J Headache Pain, $22(1), 25$. https://doi.org/10.1186/s10194-021-01240-8.

15. Kim, J., Criaud, M., Cho, S. S., Diez-Cirarda, M., Mihaescu, A., Coakeley, S., . . Strafella, A. P. (2017). Abnormal intrinsic brain functional network dynamics in Parkinson's disease. Brain, 140(11), 2955-2967. https://doi.org/10.1093/brain/awx233.

16. Kim, Y. E., Kim, M. K., Suh, S. I., \& Kim, J. H. (2021). Altered trigeminothalamic spontaneous low-frequency oscillations in migraine without aura: a resting-state fMRI study. BMC Neurol, 21(1), 342. https://doi.org/10.1186/s12883-021-02374-7.

17. Kong, J., Tu, P. C., Zyloney, C., \& Su, T. P. (2010). Intrinsic functional connectivity of the periaqueductal gray, a resting fMRI study. Behav Brain Res, 211(2), 215-219. https://doi.org/10.1016/j.bbr.2010.03.042.

18. Leonardi, M., Steiner, T. J., Scher, A. T., \& Lipton, R. B. (2005). The global burden of migraine: measuring disability in headache disorders with WHO's Classification of Functioning, Disability and Health (ICF). J Headache Pain, 6(6), 429-440. https://doi.org/10.1007/s10194-005-0252-4.

19. Li, J., Duan, X., Cui, Q., Chen, H., \& Liao, W. (2019). More than just statics: temporal dynamics of intrinsic brain activity predicts the suicidal ideation in depressed patients. Psychol Med, 49(5), 852-860. https://doi.org/10.1017/S0033291718001502.

20. Li, Z., Lan, L., Zeng, F., Makris, N., Hwang, J., Guo, T., . . Kong, J. (2017). The altered right frontoparietal network functional connectivity in migraine and the modulation effect of treatment. Cephalalgia, 37(2), 161-176. https://doi.org/10.1177/0333102416641665.

21. Li, Z., Liu, M., Lan, L., Zeng, F., Makris, N., Liang, Y., . . Kong, J. (2016). Altered periaqueductal gray resting state functional connectivity in migraine and the modulation effect of treatment. Sci Rep, 6, 20298. https://doi.org/10.1038/srep20298.

22. Li, Z., Zeng, F., Yin, T., Lan, L., Makris, N., Jorgenson, K., . . Kong, J. (2017). Acupuncture modulates the abnormal brainstem activity in migraine without aura patients. Neuroimage Clin, 15, 367-375. https://doi.org/10.1016/j.nicl.2017.05.013.

23. Liao, W., Li, J., Ji, G. J., Wu, G. R., Long, Z., Xu, Q., . . Chen, H. (2019). Endless Fluctuations: Temporal Dynamics of the Amplitude of Low Frequency Fluctuations. IEEE Trans Med Imaging, 38(11), 2523-2532. https://doi.org/10.1109/TMI.2019.2904555.

24. Liu, L., Tian, T., Li, X., Wang, Y., Xu, T., Ni, X., ... Zhao, L. (2021). Revealing the Neural Mechanism Underlying the Effects of Acupuncture on Migraine: A Systematic Review. Front Neurosci, 15, 674852. https://doi.org/10.3389/fnins.2021.674852.

25. Liu, S., Luo, S., Yan, T., Ma, W., Wei, X., Chen, Y., . . Wang, B. (2021). Differential Modulating Effect of Acupuncture in Patients With Migraine Without Aura: A Resting Functional Magnetic Resonance Study. Front Neurol, 12, 680896. https://doi.org/10.3389/fneur.2021.680896.

26. Meier, J. D., Aflalo, T. N., Kastner, S., \& Graziano, M. S. (2008). Complex organization of human primary motor cortex: a highresolution fMRI study. J Neurophysiol, 100(4), 1800-1812. https://doi.org/10.1152/jn.90531.2008.

27. Parvizi, J., Jacques, C., Foster, B. L., Witthoft, N., Rangarajan, V., Weiner, K. S., \& Grill-Spector, K. (2012). Electrical stimulation of human fusiform face-selective regions distorts face perception. J Neurosci, 32(43), 14915-14920. https://doi.org/10.1523/JNEUROSCI.2609-12.2012.

28. Schwedt, T. J., Chiang, C.-C., Chong, C. D., \& Dodick, D. W. (2015). Functional MRI of migraine. The Lancet Neurology, 14(1), 81-91. https://doi.org/10.1016/s1474-4422(14)70193-0.

29. Tian, Z., Guo, Y., Yin, T., Xiao, Q., Ha, G., Chen, J., . . Zeng, F. (2021). Acupuncture Modulation Effect on Pain Processing Patterns in Patients With Migraine Without Aura. Front Neurosci, 15, 729218. https://doi.org/10.3389/fnins.2021.729218.

30. Tian, Z., Yin, T., Xiao, Q., Dong, X., Yang, Y., Wang, M., .. . Lan, L. (2021). The Altered Functional Connectivity With Pain Features Integration and Interaction in Migraine Without Aura. Front Neurosci, 15, 646538. https://doi.org/10.3389/fnins.2021.646538.

Page $11 / 16$ 
31. Tu, Y., Fu, Z., Zeng, F., Maleki, N., Lan, L., Li, Z., . . Kong, J. (2019). Abnormal thalamocortical network dynamics in migraine. Neurology, 92(23), e2706-e2716. https://doi.org/10.1212/wnl.0000000000007607.

32. Tu, Y., Zeng, F., Lan, L., Li, Z., Maleki, N., Liu, B., . . Kong, J. (2020). An fMRI-based neural marker for migraine without aura. Neurology, 94(7), e741-e751. https://doi.org/10.1212/WNL.0000000000008962.

33. Ueda, R., Yanagisawa, K., Ashida, H., \& Abe, N. (2018). Executive control and faithfulness: only long-term romantic relationships require prefrontal control. Exp Brain Res, 236(3), 821-828. https://doi.org/10.1007/s00221-018-5181-y.

34. Wang, J. J., Chen, X., Sah, S. K., Zeng, C., Li, Y. M., Li, N., . . Du, S. L. (2016). Amplitude of low-frequency fluctuation (ALFF) and fractional ALFF in migraine patients: a resting-state functional MRI study. Clin Radiol, 71(6), 558-564. https://doi.org/10.1016/j.crad.2016.03.004.

35. Wei, H. L., Chen, J., Chen, Y. C., Yu, Y. S., Guo, X., Zhou, G. P., . . Zhang, H. (2020). Impaired effective functional connectivity of the sensorimotor network in interictal episodic migraineurs without aura. $J$ Headache Pain, 21(1), 111. https://doi.org/10.1186/s10194-020-01176-5.

36. Xu, S., Yu, L., Luo, X., Wang, M., Chen, G., Zhang, Q., .. Wang, W. (2020). Manual acupuncture versus sham acupuncture and usual care for prophylaxis of episodic migraine without aura: multicentre, randomised clinical trial. BMJ, $368, \mathrm{~m} 697$. https://doi.org/10.1136/bmj.m697.

37. Yang, S., Meng, Y., Li, J., Fan, Y. S., Du, L., Chen, H., \& Liao, W. (2019). Temporal dynamic changes of intrinsic brain activity in schizophrenia with cigarette smoking. Schizophr Res, 210, 66-72. https://doi.org/10.1016/j.schres.2019.06.012.

38. Zang, Y. F., He, Y., Zhu, C. Z., Cao, Q. J., Sui, M. Q., Liang, M., . . Wang, Y. F. (2007). Altered baseline brain activity in children with ADHD revealed by resting-state functional MRI. Brain Dev, 29(2), 83-91. https://doi.org/10.1016/j.braindev.2006.07.002

39. Zhao, L., Chen, J., Li, Y., Sun, X., Chang, X., Zheng, H., . . Liang, F. (2017). The Long-term Effect of Acupuncture for Migraine Prophylaxis: A Randomized Clinical Trial. JAMA Intern Med, 177(4), 508-515. https://doi.org/10.1001/jamainternmed.2016.9378.

40. Zhao, L., Liu, J., Zhang, F., Dong, X., Peng, Y., Qin, W., . . Liang, F. (2014). Effects of long-term acupuncture treatment on restingstate brain activity in migraine patients: a randomized controlled trial on active acupoints and inactive acupoints. PLoS One, 9(6), e99538. https://doi.org/10.1371/journal.pone.0099538.

41. Zhu, F., Liu, F., Guo, W., Chen, J., Su, Q., Zhang, Z., . . Zhao, J. (2018). Disrupted asymmetry of inter- and intra-hemispheric functional connectivity in patients with drug-naive, first-episode schizophrenia and their unaffected siblings. EBioMedicine, 36, 429-435. https://doi.org/10.1016/j.ebiom.2018.09.012.

42. Zou, Y., Tang, W., Li, X., Xu, M., \& Li, J. (2019). Acupuncture Reversible Effects on Altered Default Mode Network of Chronic Migraine Accompanied with Clinical Symptom Relief. Neural Plast, 2019, 5047463. https://doi.org/10.1155/2019/5047463.

\section{Figures}




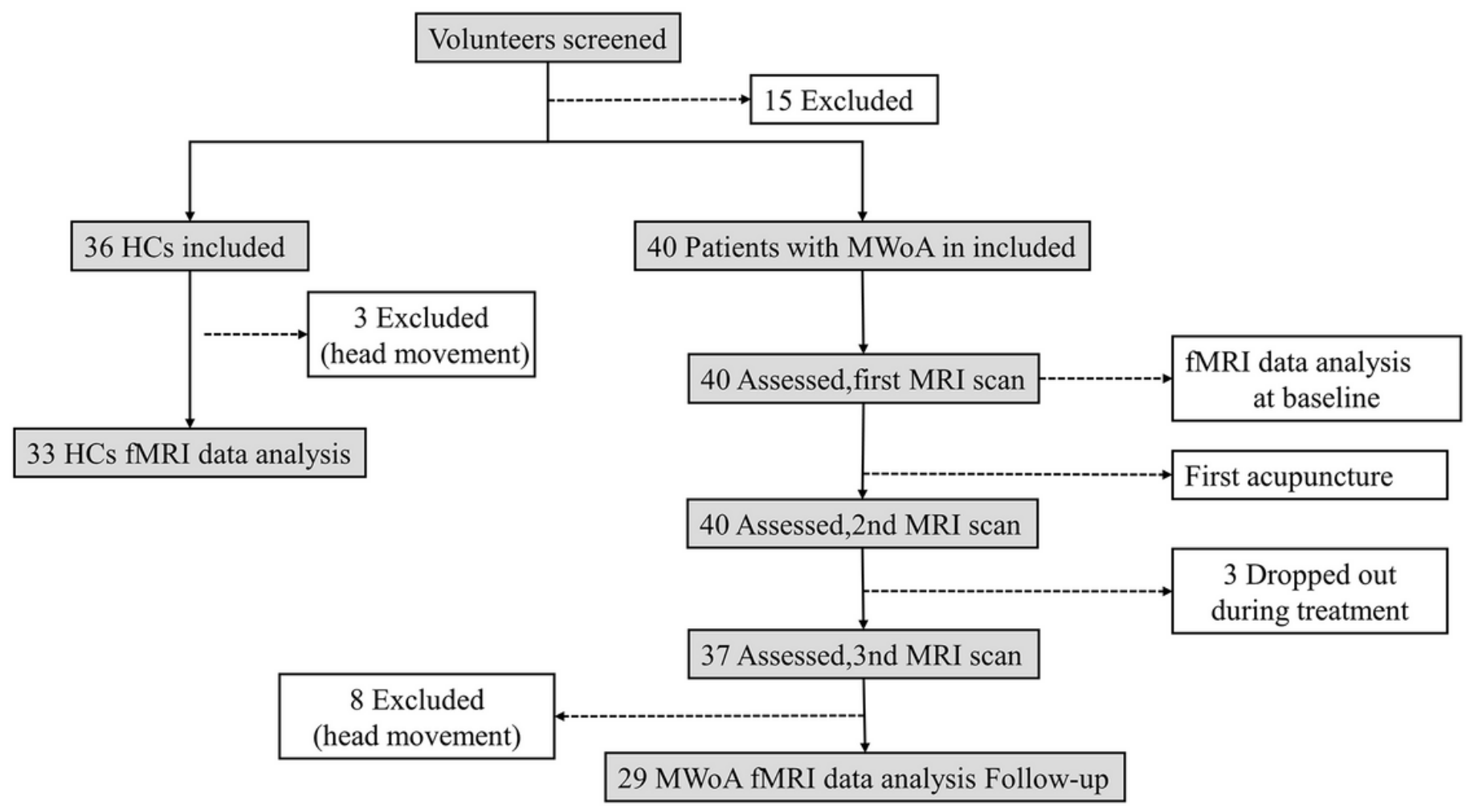

Figure 1

Flow chart displaying the screening, enrolment and treatment. All abbreviations are defined in the Abbreviations section.

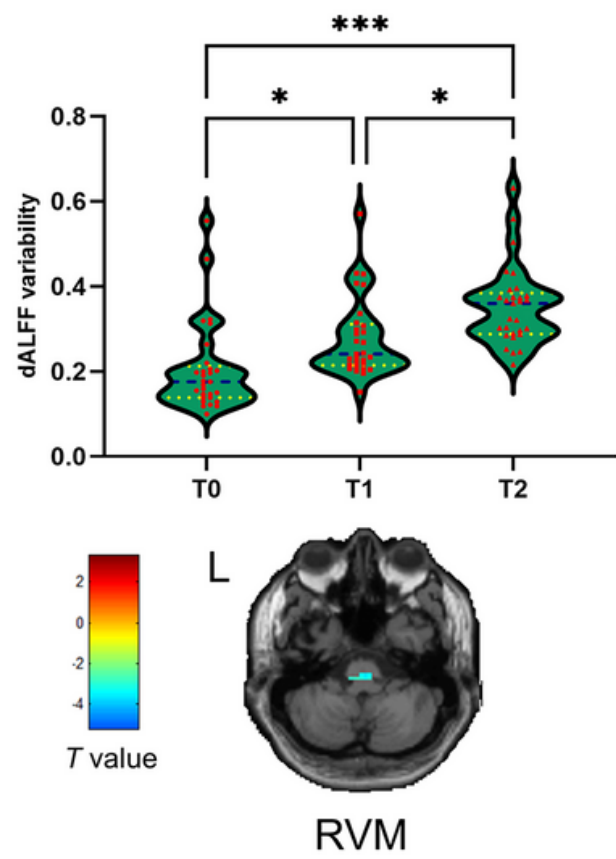

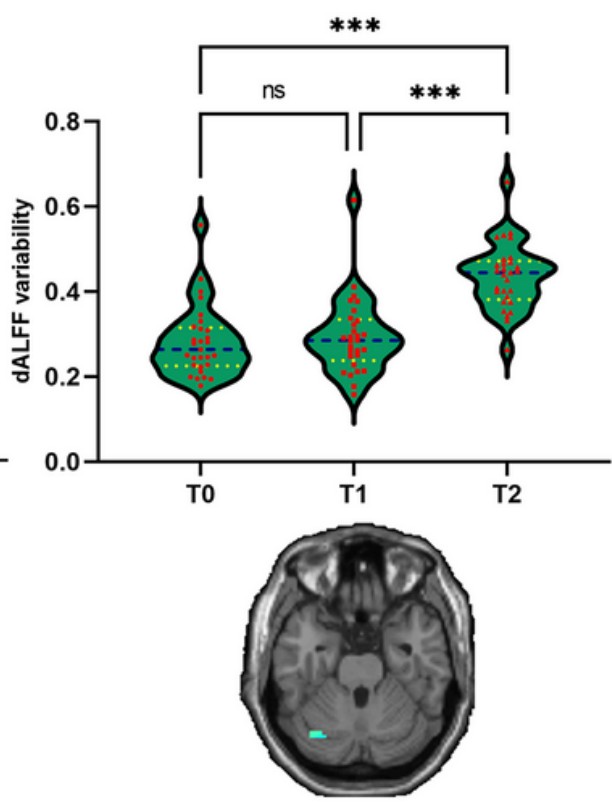

Cerebelum_Crus1_L

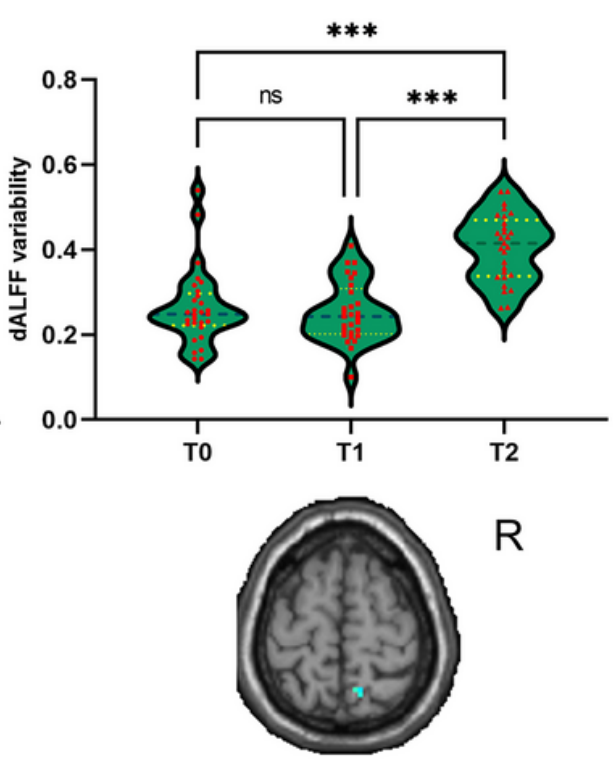

PCUN.R

Figure 2 
The dALFF variability significantly during the different periods of treatment. ${ }^{*}$ means $p<0.05$; ${ }^{\star \star \star}$ means $p<0.001$. Cool color represents decreased dALFF variability values. T0: the time point at baseline; T1: the time point after the first acupuncture session; T2: the time point after the 12th acupuncture sessions. All abbreviations are defined in the Abbreviations section.

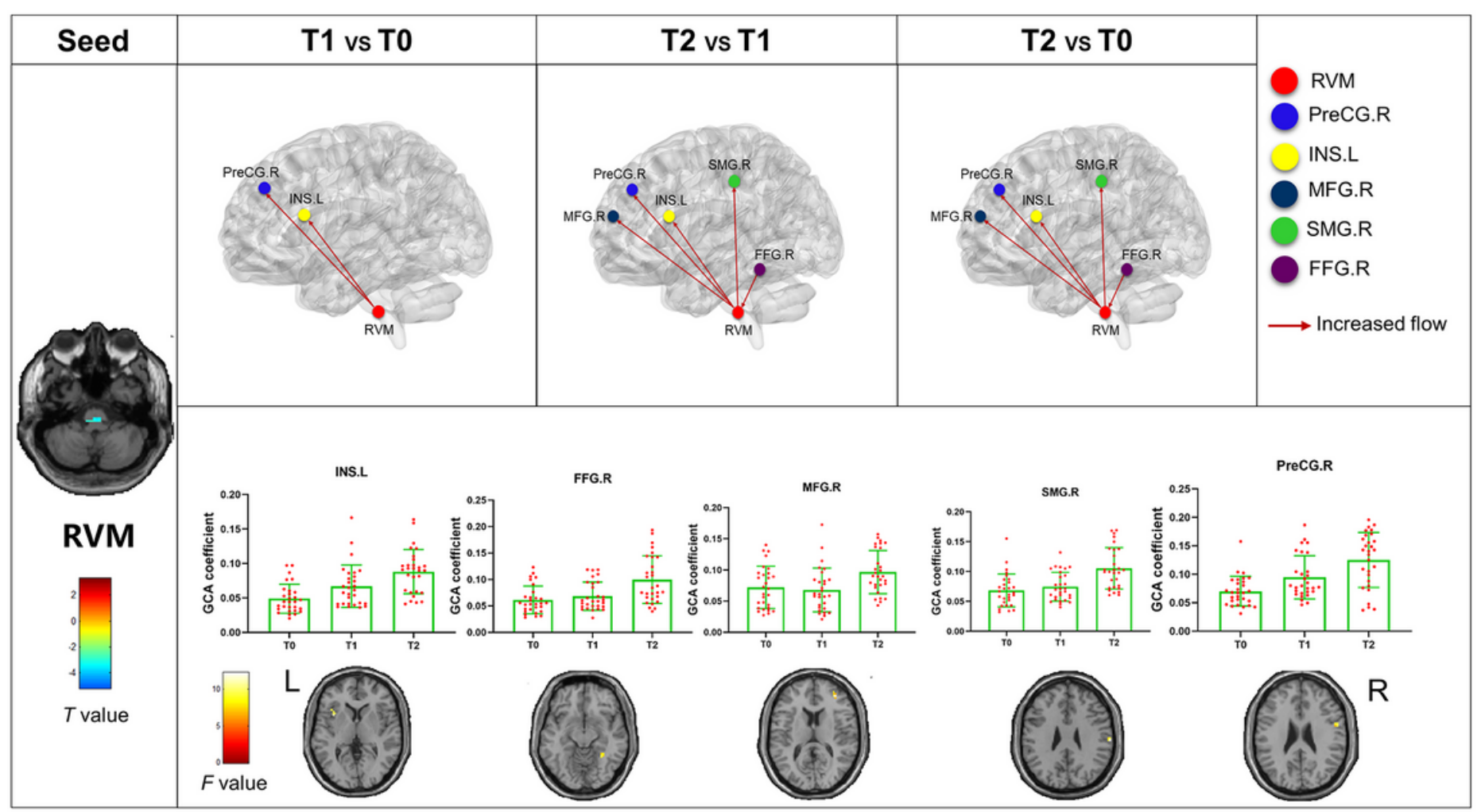

Figure 3

Abnormal effective connectivity pathways associated with the RVM. Each bar chart reflects the values of dynamic GCA coefficient in the corresponding group. Fuchsia arrows indicate significantly enhanced DEC variability. T0: the time point at baseline; T1: the time point after the first acupuncture session; T2: the time point after the 12th acupuncture sessions. All abbreviations are defined in the Abbreviations section. 


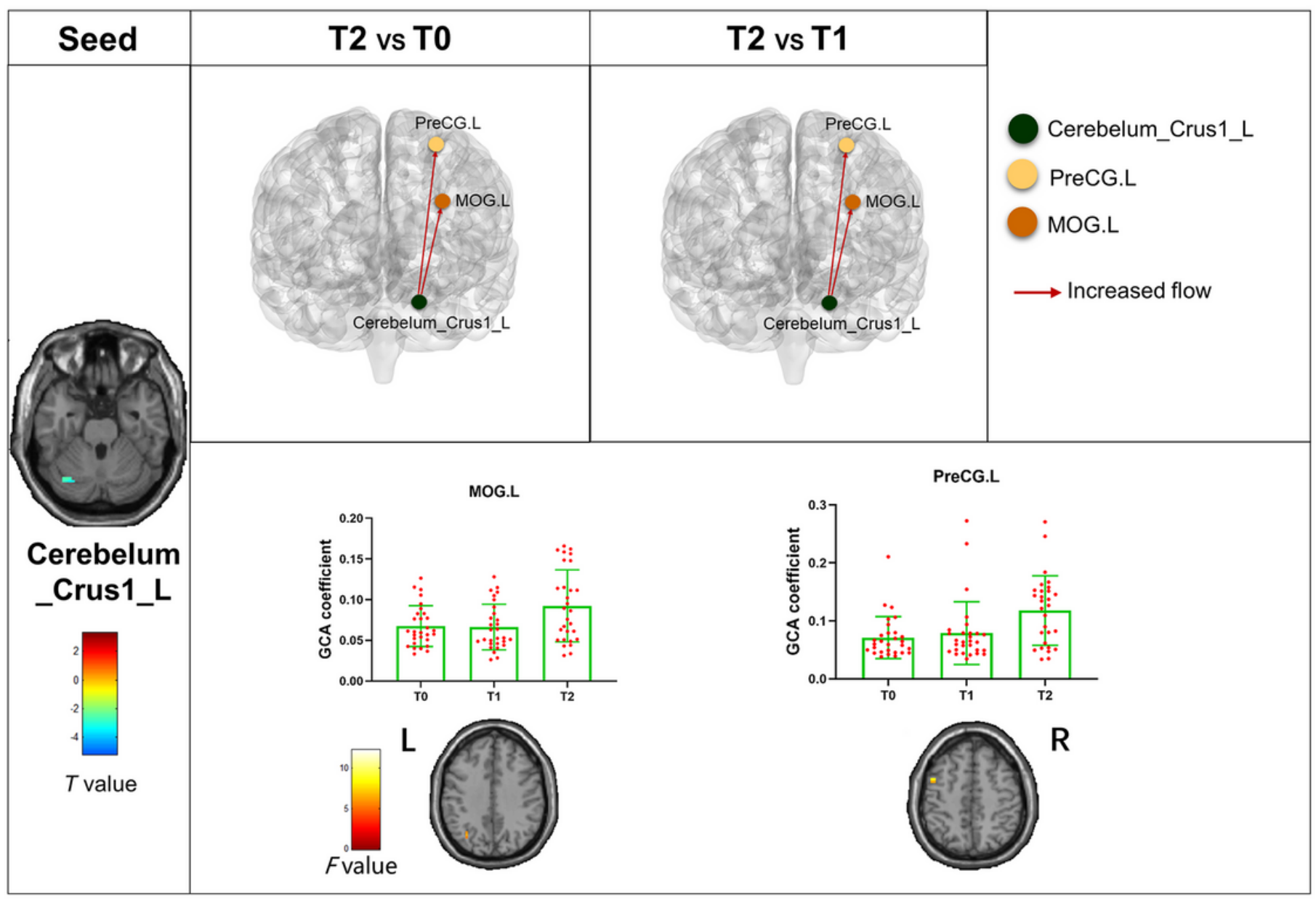

Figure 4

Abnormal effective connectivity pathways associated with the Cerebelum_Crus1_L. Each bar chart reflects the values of dynamic GCA coefficient in the corresponding group. Fuchsia arrows indicate significantly enhanced DEC variability. TO: the time point at baseline; T1: the time point after the first acupuncture session; T2: the time point after the 12th acupuncture sessions. All abbreviations are defined in the Abbreviations section. 


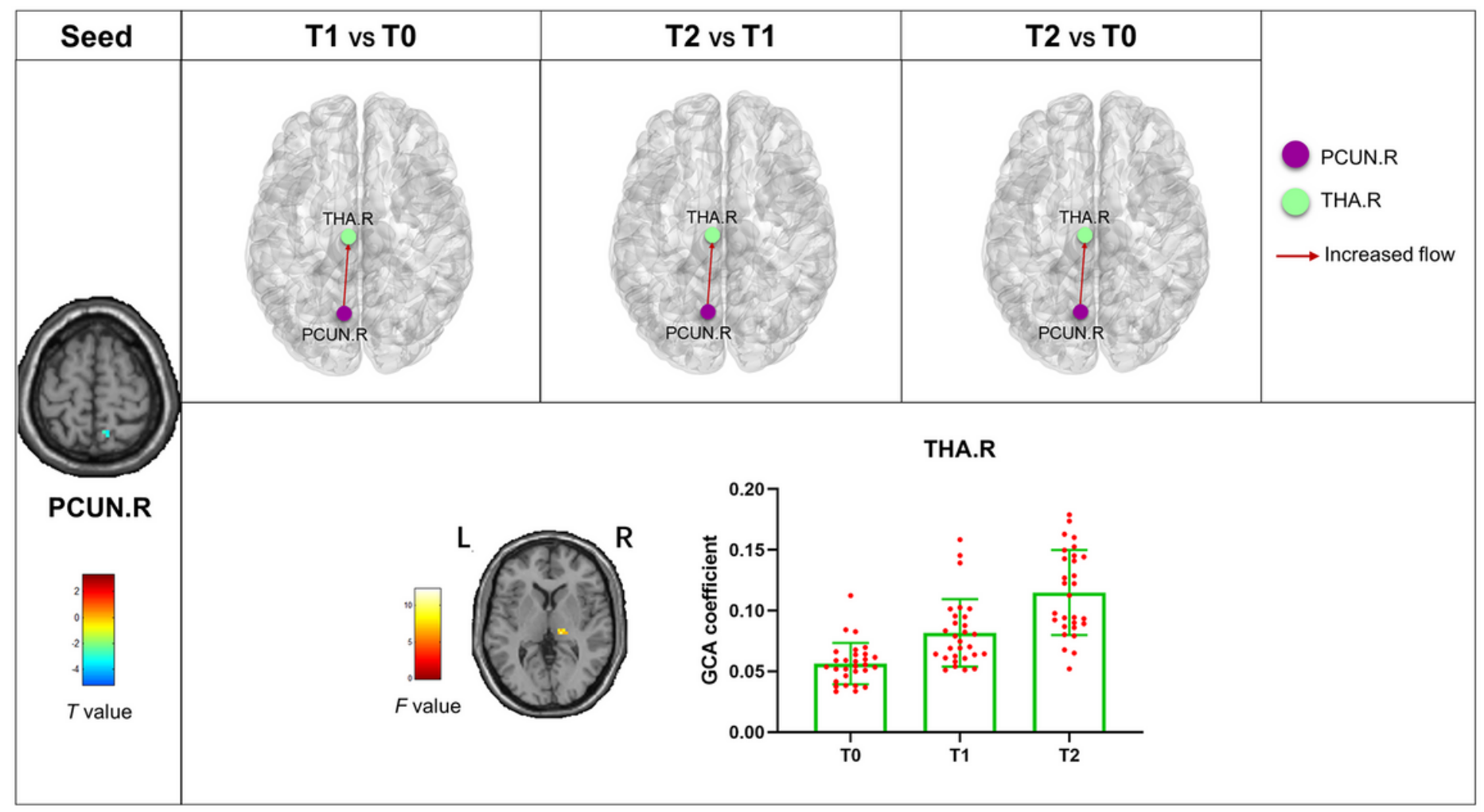

\section{Figure 5}

Abnormal effective connectivity pathways associated with the PCUN.R. Each bar chart reflects the values of dynamic GCA coefficient in the corresponding group. Fuchsia arrows indicate significantly enhanced DEC variability. T0: the time point at baseline; T1: the time point after the first acupuncture session; T2: the time point after the 12th acupuncture sessions. All abbreviations are defined in the Abbreviations section.

\section{Supplementary Files}

This is a list of supplementary files associated with this preprint. Click to download.

- Supplementarymaterials.docx 